\title{
Attenuation of the Muscle Metaboreflex in Patients with Type 2 Diabetes
}

\author{
Toshihiro Kawae, RPT, MS ${ }^{1)}$, Makoto Takahashi, RPT, PhD ${ }^{2)}$, Kana Konishi, RPT ${ }^{2)}$, \\ Kiyokazu Sekikawa, RPT, PhD ${ }^{2)}$, Tsutomu Inamizu, MD, $\mathrm{PhD}^{2}$, \\ Fukashi Ishibashi, MD, $\mathrm{PhD}^{3)}$, Yoshihiro Ito, $\mathrm{RPT}, \mathrm{MS}^{1)}$, \\ Hiroaki Kimura, MD, $\mathrm{PhD}^{4}$, Hironobu Hamada, MD, $\mathrm{PhD}^{2)}$ \\ 1) Division of Rehabilitation, Clinical Support Department, Hiroshima University Hospital \\ 2) Graduate School of Health Sciences, Hiroshima University: 1-2-3 Kasumi, Minami-ku, Hiroshima \\ 734-8553, Japan.TEL \& FAX: +81 82-257-5426,E-mail: sekikawa@hiroshima-u.ac.jp \\ 3) Ishibashi Clinic \\ 4) Department of Rehabilitation, Hiroshima University Hospital
}

\begin{abstract}
Purpose] The aim of this study was to investigate whether the muscle metaboreflex is attenuated in subjects with type 2 diabetes. [Subjects] Ten subjects with type 2 diabetes and 10 age-matched, healthy control subjects participated in the study. [Methods] We compared cardiovascular responses between the diabetic and control subjects during a static handgrip exercise at 30\% maximal voluntary contraction, followed by periods of post-exercise ischemia. [Results] During post-exercise ischemia, mean blood pressure and total peripheral resistance were not maintained at significantly higher levels than resting values in the diabetic subjects, whereas they remained elevated in the control subjects. [Conclusion] These findings indicate that the muscle metaboreflex is attenuated in subjects with type 2 diabetes.

Key words: Muscle metaboreflex, Type 2 diabetes, Postexercise ischemia
\end{abstract}

(This article was submitted Feb. 3, 2012, and was accepted Mar. 28, 2012)

\section{INTRODUCTION}

Type 2 diabetes mellitus (DM) is an important public health problem associated with high morbidity and mortality. Exercise intolerance is a major manifestation in diabetic patients ${ }^{1-3)}$, and is related to increased mortality ${ }^{4}$. For example, maximum tolerable workload and oxygen uptake have been shown to be reduced in diabetic subjects compared with healthy subjects matched for age, weight, and physical activity level ${ }^{1-3)}$. Although the reasons for this exercise intolerance are not well understood, one potential mechanism is impaired autonomic regulation of the cardiovascular system.

During dynamic exercise, heart rate (HR), stroke volume (SV), and cardiac output (CO) increase markedly in order to increase blood flow to exercising skeletal muscles ${ }^{5)}$. Cardiac output is the major limiting factor for oxygen uptake during dynamic exercise ${ }^{5)}$, and there is evidence that subjects with diabetes have impaired $\mathrm{HR}, \mathrm{SV}$, and $\mathrm{CO}$ responses to increasing workload during dynamic exercise ${ }^{6,7)}$.

It has been reported that performance of static (isometric) exercises is impaired in diabetic subjects ${ }^{8)}$. Unlike dynamic exercise, blood flow to the exercising muscle is decreased during static exercise due to a large increase in intramuscular pressure $^{5)}$. In order to maintain adequate perfusion of the exercising muscle under these conditions, it is necessary to significantly increase blood pressure (BP) by sympa- thetic vasoconstriction rather than increase of $\mathrm{CO}^{5)}$. There is evidence that BP increases are lower in diabetic subjects during static exercise than in healthy subjects ${ }^{9}$, indicating that impaired BP control may lower performance capacity during static exercise. However, to date no study has evaluated the neural mechanisms responsible for impaired BP control during static exercise performed by subjects with type 2 diabetes.

Two distinct neural control mechanisms are activated during exercise: a feed-forward neural drive from higher brain centers (termed central command), and a feedback peripheral drive from group III (predominantly mechanically sensitive) and group IV (predominantly metabolically sensitive) afferent skeletal muscles (termed the exercise pressor reflex $)^{5)}$. Given their essential roles in cardiovascular regulation, both of these inputs are potential candidates for the impaired BP response to static exercise observed in subjects with type $2 \mathrm{DM}$. Of these neural inputs, the metabolically sensitive component of the reflex, termed the metaboreflex, is responsible primarily for exercise-induced sympathoexcitation, leading to an increase in systemic vascular resistance and subsequent elevation of $\mathrm{BP}^{5)}$. We therefore hypothesized that impaired BP control during static exercise in subjects with type 2 DM may be caused by an attenuated metaboreflex.

Post-exercise ischemia (PEI), the trappping of metabolites produced during static exercise, provides a means of 
Table 1. Demographic and clinical characteristics of the diabetic and control groups

\begin{tabular}{lcc}
\hline & DM & Control \\
\hline Gender (Male/Female) & $8 / 2$ & $4 / 6$ \\
Age $(\mathrm{yrs})$ & $64.6 \pm 1.1$ & $61.6 \pm 2.1$ \\
Height $(\mathrm{cm})$ & $161.5 \pm 4.4$ & $160.2 \pm 8.1$ \\
Weight $(\mathrm{kg})$ & $57.9 \pm 6.5$ & $54.2 \pm 9.6$ \\
Body mass index $\left(\mathrm{kg} / \mathrm{m}^{2}\right)$ & $22.1 \pm 2.0$ & $20.9 \pm 0.8$ \\
Fasting blood sugar $(\mathrm{mg} / \mathrm{dl})$ & $126.3 \pm 28.4$ & - \\
Hemoglobin Alc $(\mathrm{NGSP})(\%)$ & $7.1 \pm 0.5$ & - \\
Duration of diabetes $(\mathrm{yrs}) *$ & $13.6 \pm 5.8$ & - \\
Maximal voluntary contraction $(\mathrm{kg})$ & $31.2 \pm 5.8$ & $28.3 \pm 9.1$ \\
\hline
\end{tabular}

*Years since diagnosis of diabetes mellitus.

isolating the metaboreflex from the central command and the mechanical component of the exercise pressor reflex, termed the mechanoreflex ${ }^{10,11)}$. The present study therefore compared cardiovascular responses between subjects with type $2 \mathrm{DM}$ and healthy age-matched controls during static exercise, followed by a period of PEI.

\section{SUBJECTS AND METHODS}

Ten subjects with type 2 diabetes (DM group) and 10 age-matched healthy control subjects (control group) participated in this study. The study was performed in accordance with the Declaration of Helsinki and was approved by the ethics committee of Hiroshima University Graduate School of Health Sciences (\#0904). The experimental protocols and procedures were well explained in advance to all the study participants, who then provided their written, informed consent. The characteristics of the subjects are summarized in Table 1. Subjects were excluded if they had unstable cardiovascular disease or respiratory disease, or a neurological or musculoskeletal disorder. None of the subjects were taking beta-blockers or angiotensin-II receptor blockers.

Before the experimental session, the subjects were instructed to avoid alcohol, caffeine, and strenuous physical activity for $24 \mathrm{~h}$. The subjects were seated comfortably with a handgrip dynamometer (MLT003/D, AD Instruments) held in their right hand with the arm supported. Maximal voluntary contraction (MVC) was determined as the highest force generated in several attempts at maximal effort. After a 2-min rest period, each subject performed $2 \mathrm{~min}$ of isometric handgrip (HG) at $30 \% \mathrm{MVC}$, followed by $2 \mathrm{~min}$ of forearm ischemia to isolate the muscle metaboreflex (PEI). The handgrip force exerted was recorded continuously and displayed on a computer monitor to provide visual feedback (Chart v5.0 and Powerlab, AD Instruments). PEI was achieved by inflating an occlusion cuff, positioned around the exercising arm above the elbow, to suprasystolic pressure $(>200 \mathrm{mmHg}$ ) immediately prior to the end of the handgrip exercise (E20Rapid cuff inflator, Hokanson Inc). During this exercise, the subjects were instructed not to stop breathing in order to avoid Valsalva-like maneuvers.

Arterial BP was measured continuously on a beat-by-beat basis on the middle finger of the non-exercising hand using servocontrolled finger photoplethysmography (Portapres model 2, TNO-Biomedical Instrumentation). Mean BP $(\mathrm{MBP})$ was calculated as: $\mathrm{MBP}=$ diastolic $\mathrm{BP}(\mathrm{DBP})+1 / 3$ [systolic BP (SBP) - DBP]. HR was measured using a threelead electrocardiogram (Dynascope-3140, Fukuda Densi). SV was estimated by the Modelflow method (Beatscope software v1.0, TNO-Biomedical Instrumentation), which computes aortic blood flow from arterial pressure waves by simulating a nonlinear, time-variable, three-element model of aortic input impedance ${ }^{12)}$. CO was calculated by multiplying SV by HR, and TPR by dividing MBP by CO. All the data were fed simultaneously to a computer at a sampling frequency of $1 \mathrm{kHz}$ (Powerlab, AD Instruments) for off-line analysis.

The baseline values of the cardiovascular variables during the resting state were determined as the mean over the $60 \mathrm{~s}$ prior to the start of exercise. All values were then expressed as relative changes from the resting baseline. Mean values during handgrip exercise (HG) and PEI were calculated for each 2-min period.

Group differences in the characteristics and cardiovascular values of the subjects under each condition (rest, HG and PEI) were compared using the unpaired t-tests. Dunnett's multiple comparison test (rest vs. HG and PEI) was used to compare cardiovascular values under the three conditions. The data are expressed as means $\pm \mathrm{SE}$, and the level of statistical significance was defined as $p<0.05$. The analyses were carried out using SPSS v12.0 J (SPSS Inc.).

\section{RESULTS}

There were no significant differences between the two groups in age, height, weight, or BMI (Table 1). MVC was not different between the two groups either (Table 1).

The resting baseline cardiovascular values before exercise are summarized in Table 2. The baseline values of SBP and MBP in the DM subjects were significantly higher than those of the control subjects $(\mathrm{p}<0.05)$. The other cardiovascular values were not significantly different between the two groups.

The relative changes in cardiovascular values during 
HG exercise and PEI from rest are summarized in Table 3. During HG exercise, MBP, HR, SV, CO, and TPR were significantly increased above their resting levels in both groups $(\mathrm{p}<0.05)$. The changes in all the cardiovascular values from rest were not significantly different between the two groups.

During PEI, MBP and TPR remained significantly elevated above therir baseline values in the control subjects $(\mathrm{p}<0.05)$, but not in the DM subjects. On the other hand, $\mathrm{HR}, \mathrm{SV}$ and CO during PEI were not significantly different from their baseline values in either group. There was no difference between the two groups in any of the cardiovascular parameters during PEI.

\section{DISCUSSION}

To the best of our knowledge this is the first paper to show that MBP and TRR are not elevated above resting levels during PEI in patients with type 2 DM, whereas both variables were increased in control subjects under the same condition. As we showed there were no differences in MBP responses during HG exercise between diabetic and control subjects, our data indicates that these inadequate responses in patients with type $2 \mathrm{DM}$ may be caused by an attenuated muscle metaboreflex. This finding also suggests that the central command and/or muscular mechanoreflex is enhanced in order to compensate for the attenuated pressor response, which is probably induced by an impaired muscular metaboreflex.

The neural mechanisms of cardiovascular regulation during exercise in patients with type 2 DM remain to be clarified, particularly those related to the muscular metaboreflex. The muscular metaboreflex contributes signif-

Table 2. Baseline cardiovascular parameters of the diabetic and control groups

\begin{tabular}{lrr}
\hline & \multicolumn{1}{c}{ DM } & \multicolumn{1}{c}{ Control } \\
\hline SBP (mmHg) & $154.1 \pm 7.7$ & $129.8 \pm 5.8$ \\
DBP (mmHg) & $87.5 \pm 3.9$ & $83.4 \pm 3.9$ \\
MBP (mmHg) & $110.4 \pm 5.3$ & $98.3 \pm 3.7$ \\
HR (bpm) & $60.2 \pm 4.7$ & $62.7 \pm 1.7$ \\
SV (ml) & $83.1 \pm 6.9$ & $77.3 \pm 6.8$ \\
CO (1/min) & $5.1 \pm 1.2$ & $4.9 \pm 1.1$ \\
TPR (MU) & $1.5 \pm 0.5$ & $1.1 \pm 0.2$ \\
\hline
\end{tabular}

icantly to the regulation of the cardiovascular system during static exercise. Afferent information generated by activation of metabolically sensitive (e.g. ATP, lactate and $\mathrm{pH}$ ) skeletal muscle receptors and their associated afferent fibers (group IV) is processed within the nucleus tractus solitarius (NTS) of the brainstem which reflexively adjusts sympathetic nerve activity ${ }^{11)}$. In this study, we used PEI after static HG exercise to activate and evaluate the muscular metaboreflex separate from the mechanoreflex. The key procedure consisted of inflating a cuff to suprasystolic pressure proximal to the exercising muscle in order to stop arterial inflow and venous outflow after exercise. This resulted in accumulation of muscular metabolites produced by exercise and consistent activation of the metabolically sensitive afferent nerves. As muscle does not contract during PEI, neither the central command nor the muscle mechanoreflex are operating, and in consequence, the cardiovascular response is solely due to the activation of the muscular metaboreflex. It is well known that the muscular metaboreflex raises BP primarily through increasing peripheral vasoconstriction mediated by sympathetic nerves, but that it has little effect on cardiac function. Consistent with this mechanism, we showed in control subjects that MBP and TPR during PEI remained higher than at baseline, whereas HR, SV, and CO decreased towards resting levels (Table 3 ). In contrast, in patients with type $2 \mathrm{DM}, \mathrm{MBP}$ and TPR were not significantly higher than the resting levels. These results indicate that sympathoexcitation induced by the muscular metaboreflex was blunted in patients with type $2 \mathrm{DM}$, possibly resulting in an attenuated response in MBP and TPR during PEI.

Determining the mechanisms responsible for the attenuation of the muscular metaboreflex in patients with type 2 DM was beyond the scope of the present study. It was suggested recently that blunting of the metaboreflex in patients with heart failure may be the result of a chronic reduction in skeletal muscle perfusion, resulting in insufficient removal of metabolites produced during exercise ${ }^{11)}$. It is thought that chronic exposure to these excess metabolites may lead to downregulation of metaboreceptors or decrease their sensitivity ${ }^{11)}$. It is therefore possible that this chronic exposure to metabolites also occurs in patients with type $2 \mathrm{DM}$, as they are known to have reduced blood flow to the muscles both at rest ${ }^{13)}$ and during exercise ${ }^{14,15)}$. Evidence in support of this mechanism is that administration of capsaicin, a transient receptor potential vanilloid 1 (TRPV1), into a limb artery causes selective activation

Table 3. Relative changes in cardiovascular parameters during HG exercise and PEI in the diabetic and control groups

\begin{tabular}{lcccc}
\hline & \multicolumn{2}{c}{ HG exercise } & \multicolumn{2}{c}{ PEI } \\
\hline & DM & Control & DM & Control \\
\hline MBP (mmHg) & $17.2 \pm 6.6^{*}$ & $14.9 \pm 6.4^{*}$ & $10.5 \pm 11.1$ & $8.7 \pm 8.1^{*}$ \\
HR (bpm) & $6.2 \pm 4.7^{*}$ & $5.8 \pm 3.3^{*}$ & $3.2 \pm 4.7$ & $1.3 \pm 2.4$ \\
SV (ml) & $4.5 \pm 3.0^{*}$ & $4.5 \pm 3.8^{*}$ & $1.7 \pm 8.6$ & $3.3 \pm 3.7$ \\
CO (1/min) & $0.2 \pm 0.7^{*}$ & $0.2 \pm 0.1^{*}$ & $0.1 \pm 0.5$ & $0.1 \pm 0.1$ \\
TPR (MU) & $0.3 \pm 0.3^{*}$ & $0.2 \pm 0.1^{*}$ & $0.1 \pm 0.4$ & $0.2 \pm 0.2^{*}$ \\
\hline
\end{tabular}

${ }^{*} \mathrm{p}<0.05$ vs resting values. 
of metabolically sensitive afferent fibers involved in the muscle metaboreflex, and reduced capsaicin-induced pressor responses in a mouse model of type $2 \mathrm{DM}$ was shown to be closely related to reduced vascular TRPV1 expression ${ }^{16)}$. Taken together, these findings indicate that decreased sensitivity of metabolically sensitive afferent neurons in patients with type $2 \mathrm{DM}$ may reduce metaboreflex-induced sympathoexcitation, subsequently leading to attenuated responses in MBP and TPR.

Alternatively, it is possible that alterations in muscle sympathetic nervous activity (MSNA) in an efferent route of the metaboreflex may potentially affect MBP and TPR responses. It has been shown that MSNA levels at rest influence the magnitude of the sympathetic response to physiological stress ${ }^{17)}$, suggesting that the potential for maximal sympathoexcitation diminishes with higher resting activity. As patients with type $2 \mathrm{DM}$ have been reported to have higher basal levels of MSNA ${ }^{18)}$, it is possible that these higher resting levels may limit their response to stimuli, leading to decreased peripheral vasoconstriction and subsequent limited elevation of BP.

Contrary to our initial hypothesis and the results of a previous study ${ }^{9}$, we observed that the MBP response during static $\mathrm{HG}$ exercise in patients with type $2 \mathrm{DM}$ was comparable to that in control subjects, despite the attenuation in the muscle metaboreflex. Although the reason for this discrepancy is unclear, it is possible that the central command and/or the muscle mechanoreflex may be exaggerated to compensate for the attenuated muscle metaboreflex-induced pressor response. Evidence of redundancy in the neural cardiovascular regulation system ${ }^{19)}$ suggests it is likely that attenuaton/exaggeration of one neural mechanism may modify the function of other mechanisms to adjust autonomic nerve activity and to meet the metabolic demands of working muscles during exercise. However, the complex interactions between neural cardiovascular mechanisms remain to be determined in patients with type $2 \mathrm{DM}$. As exercise plays a pivotal role in the management of type $2 \mathrm{DM}$, clarification of the regulatory mechanisms responsible for the altered cardiovascular responses to exercise is important and clinically relevant, and therefore further studies on a greater number of subjects are essential.

In summary, this study demonstrated that MBP and TPR during PEI were not increased above resting levels in patients with type $2 \mathrm{DM}$, whereas they remained elevated in control subjects. We speculate that an attenuation of the muscle metaboreflex was responsible for this phenomenon in patients with type $2 \mathrm{DM}$.

\section{REFERENCES}

1) Baldi JC, Aoina JL, Oxenham HC, et al.: Reduced exercise arteriovenous O2 difference in type 2 diabetes. J Appl Physiol, 2003, 94: 1033-1038. [Medline]

2) Regensteiner JG, Sippel J, McFarling ET, et al.: Effects of non-insulin dependent diabetes on oxygen consumption during treadmill exercise. Med Sci Sports Exerc, 1995, 27: 661-667. [Medline]

3) Regensteiner JG, Bauer TA, Reusch JE, et al.: Abnormal oxygen uptake kinetic responses in women with type II diabetes mellitus. J Appl Physiol, 1998, 85: 310-317. [Medline]

4) Wei M, Gibbons LW, Kampert JB, et al.: Low cardiorespiratory fitness and physical inactivity as predictors of mortality in men with type 2 diabetes. Ann Intern Med, 2000, 132: 605-611. [Medline]

5) Rowell LB: Cardiovascular adjustments to isometric contractions. In: $\mathrm{Hu}-$ man cardiovascular control. New York: Oxford University Press, 1993, pp $302-325$.

6) Hilsted J, Galbo H, Christensen NJ, et al.: Haemodynamic hanges during graded exercise in patients with diabetic autonomic neuropathy. Diabetologia, 1982, 22: 318-323. [CrossRef] [Medline]

7) Roy TM, Peterson HR, Snider HL, et al.: Autonomic influence on cardiovascular performance in diabetic subjects. Am J Med, 1989, 87: 382-388. [CrossRef] [Medline]

8) Petrofsky JS, Lee S: The impact of rosiglitazone on cardiovascular responses and endurance during isometric exercise in patients with Type 2 diabetes. Med Sci Monit, 2006, 12: CR21-CR26. [Medline]

9) Ewing DJ, Irving JB, Kerr F, et al.: Cardiovascular responses to sustained handgrip in normal subjects and in patients with diabetes mellitus: a test of autonomic function. Clin Sci Mol Med, 1974, 46: 295-306. [Medline]

10) Alam M, Smirk FH: Observations in man upon a blood pressure raising reflex arising from the voluntary muscles. J Physiol, 1937, 89: 372-383. [Medline]

11) Murphy MN, Mizuno M, Mitchell JH, et al.: Cardiovascular regulation by skeletal muscle reflexes in health and disease. Am J Physiol Heart Circ Physiol, 2011, 301: H1191-H1204. [CrossRef] [Medline]

12) Wesseling KH, Jansen JR, Settels JJ, et al.: Computation of aortic flow from pressure in humans using a nonlinear, three-element model. J Appl Physiol, 1993, 74: 2566-2573. [Medline]

13) Regensteiner JG, Popylisen S, Bauer TA, et al.: Oral L-arginine and vitamins $\mathrm{E}$ and $\mathrm{C}$ improve endothelial function in women with type 2 diabetes. Vasc Med, 2003, 8: 169-175. [CrossRef] [Medline]

14) Kingwell BA, Formosa M, Muhlmann M, et al.: Type 2 diabetic individuals have impaired leg blood flow responses to exercise: role of endotheliumdependent vasodilation. Diabetes Care, 2003, 26: 899-904. [CrossRef] [Medline]

15) Lalande S, Gusso S, Hofman PL, et al.: Reduced leg blood flow during submaximal exercise in type 2 diabetes. Med Sci Sports Exerc, 2008, 40: 612-617. [CrossRef] [Medline]

16) Ohanyan VA, Guarini G, Thodeti CK, et al.: Endothelin-mediated in vivo pressor responses following TRPV1 activation. Am J Physiol Heart Circ Physiol, 2011, 301: H1135-H1142. [CrossRef] [Medline]

17) Schobel HP, Oren RM, Mark AL, et al.: Influence of resting sympathetic activity on reflex sympathetic responses in normal man. Clin Auton Res, 1995, 5: 71-80. [CrossRef] [Medline]

18) Huggett RJ, Scott EM, Gilbey SG, et al.: Impact of type 2 diabetes mellitus on sympathetic neural mechanisms in hypertension. Circulation, 2003, 108: 3097-3101. [CrossRef] [Medline]

19) Mitchell JH: J.B. Wolffe memorial lecture. Neural control of the circulation during exercise. Med Sci Sports Exerc, 1990, 22: 141-154. [Medline] 